\title{
Revisión: fibroína de seda y sus potenciales aplicaciones en empaques biodegradables para alimentos
}

\section{Review: silk fibroin and their potential applications on biodegradable food packaging}

\author{
Ángel Daniel Ríos ${ }^{1}$, Catalina Álvarez López ${ }^{2}$, Luis Javier Cruz Riaño ${ }^{3}$, Adriana Restrepo Osorio ${ }^{4}$ \\ ${ }^{1}$ Ingeniero Agroindustrial, Grupo de Investigación sobre Nuevos Materiales GINUMA, \\ Universidad Pontifica Bolivariana, Medellín, Colombia \\ ${ }^{2}$ Ph. D. Ingeniera Agroindustrial, Grupo de Investigaciones Agroindustriales GRAIN, \\ Universidad Pontificia Bolivariana, Medellín, Colombia \\ ${ }^{3}$ Ph. D. Ingeniero Mecánico, Grupo de Investigación sobre Nuevos Materiales GINUMA, \\ Universidad Pontificia Bolivariana, Medellín, Colombia \\ ${ }^{4} \mathrm{Ph}$. D. Ingeniera Textil, Grupo de Investigación sobre Nuevos Materiales GINUMA, \\ Semillero de Investigación en Textiles SI Textil, Universidad Pontificia Bolivariana, Medellín, Colombia \\ E-mail:angeldaniel.rios@upb.edu.co,adriana.restrepo@upb.edu.co
}

\section{RESUMEN}

El cuidado del medioambiente y el uso responsable de los recursos, ha promovido investigaciones con diferentes biopolímeros que permitan el desarrollo de nuevos materiales que puedan ser empleados en empaques para alimentos. Entre estos biopolímeros se encuentra la fibroína de seda (FS), que representa cerca del 70\% de la fibra de seda y puede ser obtenida a partir de capullos de primera calidad o de los desperdicios generados en la producción serícola. En el desarrollo de empaques de alimentos con FS, se pueden dar dos alternativas de producción, una en la que la FS puede ser funcionalizada por otros componentes, y otra en la que se incluye la FS como componente funcional modificando otros biopolímeros. La FS puede conferirle al material de empaque ciertas propiedades, como: aumento de la permeabilidad de oxígeno, resistencia a la rotura y una mayor velocidad de degradación del biopolímero. Este artículo se centra en cuatro temas principales: empaques, biopolímeros en empaques, FS y finalmente, la FS en empaques para alimentos.

Palabras clave: Empaques, Materiales poliméricos, Biopolímeros, Fibroína de seda, Biomateriales.

\begin{abstract}
Environmental protection and responsible use of resources, have promoted research of different biopolymers allowing to develop biodegradable materials which can be used in food packaging. Among these biopolymers is silk fibroin (SF), this protein represents nearly $70 \%$ of the silk fiber and it can be obtained from high quality cocoons or from waste generated in the silk industry. In the development of food packaging with SF, there are two possible alternative of production, one in what the SF can be functionalized by other components and another in which, the FS is included as functional component modifying other biopolymers. In the case of food packaging, the SF can concede desirable properties to the package such as an increase in oxygen permeability, more tensile strength and a higher biopolymer degradation rate. This paper is focused on four main topics: packaging, biopolymers in packaging, SF and finally, the $\mathrm{SF}$ in food packaging.
\end{abstract}

Keywords: Packaging, Polymeric materials, Biopolymers, Silk fibroin, Biomaterials. 


\section{INTRODUCCIÓN}

En la actualidad, factores como el crecimiento demográfico, el aumento del poder adquisitivo y cambios en los hábitos de consumo, han provocado un aumento en el uso de empaques plásticos no biodegradables [1], lo que ha generado una problemática mundial en el manejo de los residuos sólidos que se producen, que en su mayoría se ubican en rellenos sanitarios, se arrojan a las calles o en fuentes hídricas [2]. Por tal razón, es necesario dar una buena disposición final a estos residuos, ya sea mediante incineración, producción de compost, aislándolos en lugares adecuados de manera que no representen un grave daño medio ambiental; o bien, generando valor agregado a partir de los mismos [3].

Por otra parte, dada la difícil degradación de los residuos plásticos, una alternativa más adecuada para disminuir la carga ambiental que producen los mismos, es el desarrollo de nuevos biomateriales a partir del empleo de materias primas que favorezcan su biodegradación. Algunos de estos materiales, pueden ser residuos agroindustriales cuyo aprovechamiento requiere de una evaluación de los mismos para conocer su origen, su composición y su calidad; de esta manera, se pueden definir las tecnologías más apropiadas para sus tratamientos y posteriores usos o aplicaciones [4].

En el caso de la industria serícola, en la cual se producen residuos cercanos al $80 \%$ del peso total del capullo del gusano de seda (Bombyx mori) [5], se generan subproductos fibrosos provenientes de capullos de rechazo, partes del capullo no devanables y otros desechos que no se emplean en la fabricación de productos textiles. A partir estos residuos fibrosos, es posible extraer proteínas como la fibroína [6], que puede ser usada como biomaterial en formas tan diversas como: películas, esponjas, geles o andamios celulares [7]; que son empleadas en sectores como medicina, farmacología, cosmética [8], o bien en el desarrollo de nuevos materiales que pueden reemplazar plásticos. Esto convierte a la FS en una potencial materia prima para el desarrollo de empaques o bolsas biodegradables [9].

Considerando lo anterior, en este artículo se hace una revisión bibliográfica sobre el tema de empaques y los biopolímeros empleados en esta aplicación, también se incluyen diferentes investigaciones en las que se ha empleado la FS para desarrollar biomateriales para empaques de alimentos, en algunos casos siendo la FS funcionalizada por otros componentes y en otros, incluyendo la FS como componente funcional de otros biopolímeros.

\section{EMPAQUES}

\subsection{Generalidades de los empaques}

El empaque hace relación al objeto físico que contiene un producto o alimento; se dice que es un empaque primario cuando está en contacto directo con el mismo y es un empaque secundario cuando contiene varios empaques primarios en su interior. Los empaques en general tienen cuatro funciones principales: contención, protección, comodidad y comunicación. En el caso de los alimentos, la protección ofrecida por el empaque primario, evita que factores externos como: humedad, suciedad, olores, microorganismos, fuerzas de compresión, entre otros; tengan efecto directo sobre el producto [10]. Para brindar dicha protección en los alimentos, se han utilizado una amplia variedad de materiales, entre los que se destacan: vidrio, metales, papel y plásticos [11].

\subsection{Sistemas de empaques para alimentos}

La diversidad de materiales y tecnologías desarrolladas en el sector de empaques, ha propiciado la generación de diferentes sistemas de empaques primarios para alimentos [12], entre los que sobresalen:

- Empaque en atmósferas modificadas: consiste en la inyección de ciertos gases como oxígeno, dióxido de carbono, nitrógeno o una combinación de éstos dentro del empaque antes de ser sellado, proporcionando un ambiente óptimo para aumentar la vida útil del alimento [13].

- Empaque aséptico: su funcionamiento está basado en el llenado de recipientes estériles con un producto en condiciones asépticas y sellado herméticamente, lo que implica la ausencia de microorganismos, vapor de agua y gases que puedan ingresar del ambiente [14].

- Empaque inteligente: es un sistema de empaque capaz de llevar a cabo funciones como: detección de riesgo microbiológico; registro y seguimiento de la trazabilidad del producto; incremento de la vida útil del alimento; comunicar al consumidor sobre la seguridad y la calidad del producto, con la intención de facilitar la toma de decisiones o advertir sobre posibles peligros; entre otras [15].

- Empaque activo: es aquel empaque que cambia las condiciones de los alimentos envasados para extender la vida útil o para mejorar las propiedades sensoriales, manteniendo al mismo tiempo la calidad del alimento [16].

Para el desarrollo de algunos sistemas de empaques para alimentos es frecuente el uso de diferentes polímeros. La variedad de composiciones que pueden obtenerse mediante materiales poliméricos, han relegado a materiales tradicionales (vidrio, papel y metal) en el desarrollo de materias primas para el empaque primario de alimentos [17]. 


\subsection{Materiales poliméricos en empaques}

Los polímeros son estructuras conformadas por un gran número de moléculas simples (monómeros), unidos en secuencia mediante enlaces covalentes, generalmente entre átomos de carbono. Los métodos de síntesis permiten obtener productos formados por cadenas macromoleculares de distintas longitudes. Esta distribución de pesos moleculares le confiere propiedades especiales a los polímeros que se obtienen, permitiendo la diversificación de los mismos en aplicaciones como: fabricación de plásticos, resinas, fibras sintéticas, cauchos artificiales, materiales para empaques, entre otros [18].

Las características de los materiales poliméricos dependen de las propiedades físicas y químicas del compuesto que se emplee para su elaboración; las que a su vez están determinadas por la estructura y peso molecular, grado de cristalinidad y composición química [11]. Para el empaque de alimentos se deben considerar las propiedades específicas de los polímeros, como es el caso de la porosidad, que son pequeños espacios vacíos y de diferentes tamaños dentro del material; esta propiedad depende en gran medida del método de procesamiento del polímero, y tiene influencia sobre la resistencia y la permeabilidad de gases del material [19].

Algunos polímeros se emplean en el desarrollo de empaques debido a sus propiedades especiales, como tener bajas densidades, gran flexibilidad y exhibir alta resistencia específica; además, pueden presentar una inercia química notable [20]. Entre los polímeros sintéticos que se utilizan en el desarrollo de materiales para empaques, están: el polietileno, el polipropileno, los copolímeros de etileno, las poliamidas y los poliésteres [11]. Recientemente, se ha incrementado el uso de biopolímeros en el desarrollo de empaques, como alternativas para minimizar el impacto ambiental de los materiales sintéticos [21].

\section{BIOPOLÍMEROS EN EMPAQUES}

\subsection{Generalidades de los biopolímeros}

Los biopolímeros son macromoléculas, que pueden ser obtenidos a partir de fuentes renovables como plantas, microorganismos y animales, o también es posible sintetizarlos a partir de petroquímicos, generando con esto biopolímeros que se pueden biodegradar [22]. La biodegradabilidad es la degradación del biopolímero por acción de microorganismos como hongos o bacterias, generando dióxido de carbono, metano, agua, compuestos inorgánicos o biomasa en dicho proceso. Esta capacidad de biodegradación no sólo está asociada a la fuente de origen, sino también a la estructura química de los monómeros y a las condiciones del medio ambiente, lo que a su vez genera cambios en los tiempos de degradación
$[22,23]$. Los biopolímeros biodegradables constituyen una solución al difícil manejo de los desperdicios que generan los polímeros sintéticos no degradables, ya que permiten reducir la carga ambiental [24].

\subsection{Clasificación de los biopolímeros}

Los biopolímeros pueden ser clasificados de acuerdo a su composición química, método de síntesis, importancia económica, aplicación, entre otras. Dentro de las clasificaciones que se pueden generar Michael Niaounakis, propone una de acuerdo al origen y la biodegradabilidad [23], como se indica a continuación con algunos ejemplos:
- $\quad$ Tipo A - Origen natural y biodegradables
» Polisacáridos (almidón, celulosa, lignina, quitina)
» Proteínas (gelatina, caseína, gluten de trigo, seda y lana)
" Lípidos (Triglicéridos)
» Poliésteres producidos por microorganismos y plantas (polihidroxi-alcanoatos - PHA)
» Poliésteres bioderivados de monómeros (ácido poliláctico - PLA)
» Diversos polímeros (cauchos)

- $\quad$ Tipo B - Origen natural y no biodegradables

» Poliamidas (PA 11, PA 410, PA 610, PA 1010, PA 1012)

» Poliesteramidas

» Poliésteres insaturados

» Resinas epóxicas

» Resinas fenólicas

- $\quad$ Tipo C - Origen sintético y biodegradables

» Poliésteres alifáticos (ácido poliglicólico - PGA; policaprolactona - PCL; polibutilen sucinato PBS,)

» Polivinil alcohol (PVA)

» Polialquileno dicarboxilatos (polietilen sucinato PES; polibutinel adipato - PBA)

» Polianhídridos

\subsection{Aplicaciones de biopolímeros en empaques}

Algunos biopolímeros de uso común como la celulosa y el almidón, presentan costos de producción relativamente bajos, gracias a las diferentes fuentes de las que se pueden obtener, lo que favorece la aplicación de los mismos en diversos empaques [25]. Sin embargo, otros biopolímeros se caracterizan por el bajo rendimiento de producción y los altos costos que representa elaborarlos, lo que ha limitado la aplicación de algunos de estos en ciertos sectores industriales y se ha convertido en un reto para los investigadores [22]. Entre los biopolímeros que tienen aplicación específica en empaques de alimentos, 
están los almidones y celulosas de diversas fuentes [11, 26], zeína de maíz y proteína de soja [24]; también sobresalen las mezclas entre proteína de soja con ácido poliláctico (PLA) [27], proteína de suero de leche como recubrimiento de películas de PLA [28], quitosano combinado con polímeros resistentes al agua [29], FS y PVA [30], quitosano y FS [9], entre otros.

\section{FIBROÍNA DE SEDA}

El gusano de seda (Bombyx mori) bajo condiciones adecuadas de alimentación, temperatura y humedad, puede iniciar un proceso de transformación en el que construye un capullo a manera de protección. Al interior de éste, se inicia una metamorfosis que es interrumpida por los sericultores, ya que estos capullos sirven de materia prima para la producción de la seda. Un solo capullo está formado por una fibra que puede tener más de $1500 \mathrm{~m}$ de longitud [31], ésta se compone por dos proteínas: la fibroína y la sericina; la primera tiene la forma de dos filamentos continuos, que se mantienen unidos por el recubrimiento de la sericina [32].

\subsection{Generalidades de la FS}

La FS es la principal proteína de la fibra de seda y representa entre el 70 80\% de su peso. Esta proteína, en su estructura primaria, está constituida por diferentes aminoácidos, entre los que se destacan la glicina, la alanina, la serina y la tirosina [33]. Las cuales, acorde a su peso molecular, conforman tres componentes protéicos: una proteína grande, conocida como la cadena pesada (cadena $\mathrm{H}$ ) de $350 \mathrm{kDa}$; una segunda proteína pequeña, que se conoce como cadena ligera (cadena L) de $25 \mathrm{kDa}$; y una tercera glicoproteína, llamada proteína P25 de 30 $\mathrm{kDa}$ [32]. La FS puede ser extraída directamente de las glándulas de los gusanos [31], de los capullos de primera calidad o de los subproductos fibrosos que se generan en el proceso de fabricación de textiles de la seda. Los subproductos fibrosos pueden provenir de capullos de rechazo, partes del capullo no devanables y otros productos [6].

La FS puede tener tres tipos de polimorfismos, lo que representa que la molécula puede adoptar diferentes conformaciones en su estructura cristalina [34], como son el estado glandular dentro del gusano (Seda I), la fibra excretada e hilada por el gusano, que en su mayoría se conforma por una estructura secundaria b-hoja (Seda II), y una tercera forma, que se da como resultado de factores externos generando una mayor estructura helicoidal (Seda III) [35]. Algunos tratamientos térmicos o el uso de ciertos solventes (etanol, metanol, entre otros), pueden generar cambios en la conformación estructural de la proteína [36]. Estas variaciones a su vez, producen alteraciones en las propiedades químicas y físicas de la FS, la cuales son determinadas mediante pruebas de caracterización como pruebas reológicas para determinar la viscosidad [37], análisis térmicos para evaluar el comportamiento en función de la temperatura [38], determinación del peso molecular [39] y otros análisis. De igual manera, el método de obtención de la FS, puede ser un factor que genere cambios en las estructuras de la proteína [36].

\subsection{Métodos de obtención de la FS}

Existen diferentes métodos para la obtención de la FS, todos ellos basados en la disolución de la seda en soluciones salinas altamente concentradas [40]. De acuerdo con Rockwood y su equipo de trabajo, es posible obtener la FS mediante un proceso de desengomado de la seda con carbonato de sodio $\left(\mathrm{Na}_{2} \mathrm{CO}_{3}\right)$ para eliminar la sericina y posteriormente realizando una disolución de las fibras con Bromuro de Litio ( $\mathrm{LiBr}$ ) en una concentración de 9,3 M [41]. También se puede producir FS empleando una solución de Cloruro de Calcio $\left(\mathrm{CaCl}_{2}\right)$ al $50 \%$ wt, o mediante una solución conformada por $\mathrm{CaCl}_{2}$, etanol y agua (relación: 1:8:2 moles) [42, 43].

\subsection{Aplicaciones de la FS}

LaFS puede ser transformada en diferentes presentaciones como fibras, películas, microesferas, tubos y esponjas [35]. Éstas se pueden aplicar en diversos campos, como: biomedicina [44], farmacología, óptica [8], industria cosmética [45], empaques [9] y muchos más. En cuanto a las aplicaciones relacionadas con empaques de alimentos, sobresalen el desarrollo de sensores con nanoestructuras metálicas y FS para determinar la calidad del alimento [46]; el desarrollo de empaques con inclusión de FS como componente funcional [30]; la evaluación del efecto antimicrobiano de películas comestibles de FS carragenina y extracto de semillas de uva [47]; entre otros.

\section{FIBROÍNA DE SEDA EN EMPAQUES}

\subsection{Fibroína de seda como biomaterial}

La FS es un polímero natural que presenta una serie de características de gran interés para el desarrollo de nuevos materiales, entre las que se destacan sus propiedades mecánicas y su biodegradabilidad [48, 49]. Estas características hacen que la FS pueda tener potencial aplicación como biomaterial de empaques.

Un estudio realizado por Baimark y Srihanam, tuvo como objetivo desarrollar una biopelícula a partir de una solución de FS a la que se adicionó glucosa, para mejorar la flexibilidad y la capacidad de humectación de la misma. La morfología de las películas obtenidas fue homogénea y los resultados de las pruebas de 
tracción indicaron que existe una relación directamente proporcional entre la proporción de glucosa y la resistencia a la rotura del biomaterial desarrollado. La flexibilidad que le provee la glucosa a las biopelículas de FS, brinda ciertas características mecánicas que permiten que el biomaterial elaborado pueda tener potenciales usos biomédicos, farmacéuticos y aplicaciones en el desarrollo de empaques [50].

Igualmente, se han desarrollado películas que tenían como matriz principal FS que fue mezclada con quitosano, mediante el vaciado en un recipiente y posterior evaporación del solvente. A dichas películas se les evaluó la influencia que tenía el peso molecular del quitosano (MWQ) sobre la estabilidad térmica de las mismas. La morfología del biomaterial fue observada mediante microscopía electrónica de barrido (SEM), encontrando que todas eran homogéneas; además, eran claras y transparentes sin importar el MWQ. La estabilidad térmica de las películas se vio mejorada a medida que el MWQ incrementó. Los resultados sugieren que el biomaterial desarrollado puede tener interés en campos como: biomedicina, farmacología y aplicaciones de empaques [51].

\subsection{Fibroína de seda en empaques de alimentos funcionalizada por otros componentes}

La FS puede ser combinada con algunos biopolímeros o materiales con el fin de obtener ciertas características físico químicas que permitan mejorarla; por ejemplo, su efecto antimicrobiano se ve potenciado al mezclarse con extractos de uva [47], mientras que sus propiedades físicas pueden verse beneficiadas por la inclusión de plastificantes [52] o el mejoramiento de la vida útil de alimento mediante sensores con FS y nanoestructuras metálicas.

\subsubsection{Aumento del efecto antimicrobiano}

Para mejorar el efecto antimicrobiano de recubrimientos comestibles desarrollados a partir de FS carragenina, se incorporaron extractos de semilla de uva. Dichas coberturas fueron empleadas en productos cárnicos, encontrando un aumento de la actividad antimicrobiana en los alimentos. Además, el uso de esas películas comestibles tuvo efecto significativo en el contenido de humedad y las propiedades texturales del producto alimenticio, lo que indica el potencial uso del biomaterial desarrollado como agente antimicrobiano en empaques comestibles de alimentos [47].

De igual manera, $\mathrm{Ku}$ y su equipo de trabajo elaboraron películas de FS que contenían catequina (antioxidante de origen natural) y evaluaron el efecto que tenía aumento de la concentración del antioxidante en la capacidad antimicrobiana de las mismas. Esto se pudo comprobar con salchichas inoculadas con E. coli, que fueron empacadas con la película de FS catequina y comparadas con películas de FS sin catequina. Los resultados indicaron que las películas de FS catequina redujeron la población de E. coli y aumentaron la duración del alimento [53].

\subsubsection{Cambios en algunas propiedades físicas del empaque}

A las antes mencionadas películas de FS-catequina, también se les evaluó el efecto que tenía el aumento de la concentración del antioxidante sobre algunas propiedades como la resistencia a la tracción y la permeabilidad de vapor de agua (WVP) de la película. Se encontró una relación inversa entre las propiedades evaluadas y el contenido de catequina, lo quiere decir que los valores en la resistencia a la tracción y la WVP disminuyeron con el aumento de la concentración del antioxidante [53].

De otro lado, la adición de ciertos plastificantes (sustancias incorporadas en otros materiales para aumentar su flexibilidad y facilidad de trabajo), permite mejorar propiedades físicas como la elongación, resistencia a la tracción, WVP. Materiales como polietilenglicol (PEG), polipropileno glicol (PPG) y glicerol (G) fueron combinadas con FS para evaluar dichas propiedades. Se encontró que las mezclas con $G$ presentaron mayor porcentaje de elongación, la que contenían PPG tuvieron mayor resistencia a la tracción, mientras que la inclusión del PEG disminuyó el WVP. Los resultados evidencian que estos biomateriales pueden ser aplicados en empaques de alimentos [52].

\subsubsection{Empaques inteligentes sensores}

En cuanto a aplicaciones relacionadas con empaques inteligentes de alimentos, sobresale el desarrollo de dispositivos como sensores elaborados con FS y nanoestructuras metálicas (NM). Las NM se ubican en la superficie de los alimentos y son sensibles a los cambios dieléctricos que tengan los mismos (propiedad relacionada con la composición del material y la densidad del alimento), permitiendo evaluar y controlar la evolución de éstas propiedades físico-químicas en el tiempo. Este proceso permite que el contacto entre las nanoestructuras del sensor y el alimento, proporcione un seguimiento in situ de la calidad de los alimentos que se desean consumir [46].

\subsection{Fibroína de seda en empaques de alimentos como componente funcional}

Tradicionalmente se han empleado una gran variedad de materias primas para la elaboración de empaques en 
alimentos; la selección de dichos materiales, se basa en las propiedades necesarias para la preservación y protección de un alimento en particular. Debido a sus características, la FS puede mejorar propiedades como permeabilidad de gases, resistencia mecánica, biodegradabilidad, entre otras; a los materiales para empaques de alimentos en los que ha sido incluida $[30,54]$.

\subsubsection{Mejora de la resistencia del material}

La electrohilatura es una aplicación utilizada para el desarrollo de no tejidos, nanocompuestos y nanofibras, a partir de una variedad de polímeros y biopolímeros. Las nanofibras electrohiladas se pueden emplear como refuerzo para mejorar las propiedades físicas de ciertos materiales de empaques de alimentos [55], o incluso en el diseño de empaques activos o para el empaque de alimentos nutracéuticos [56]. La FS es usada en el desarrollo de nanofibras electrohiladas con otros polímeros, mejorando sus propiedades mecánicas, una funcionalidad que tiene gran potencial en aplicaciones de empaques. Por ejemplo, no tejidos elaborados a partir de zeína y FS, presentaron un aumento en la resistencia a la tracción mientras mayor era el contenido de FS [57].

\subsubsection{Aumento de la vida útil de las frutas}

Con el fin de mejorar la vida útil de frutas en condiciones medioambientales normales, se realizaron recubrimientos con soluciones acuosas de FS, que se benefician de propiedades como la adaptabilidad, la hidrofobicidad y el polimorfismo de esta proteína. Los diferentes cambios estructurales que pueda tener la FS, permiten regular la difusión de $\mathrm{O}_{2^{\prime}} \mathrm{CO}_{2}$ y la PWV a través de las membranas. De acuerdo a Marelli y su equipo, mientras mayor es el contenido de b-hoja en la FS del recubrimiento, menor es la difusión de oxígeno a través del mismo; esto a su vez, reduce la tasa de respiración celular y la evaporación del agua de la fruta. Por lo anterior, se puede afirmar que los recubrimientos con soluciones acuosas de FS, se convierten en una prometedora alternativa para la conservación de alimentos, empleando un material de origen natural [58].

\subsubsection{Mejora la resistencia, permeabilidad de oxígeno y biodegradabilidad del empaque}

En el estudio realizado por Kuchaiyaphum y su equipo, se desarrollaron películas a partir de polivinil alcohol (PVA) mezclado con almidón de arroz en una proporción $60 / 40 \%$ (wt/wt) y adición de FS al 2\% (wt/v). Los investigadores sugieren que estas películas tienen un potencial uso en la fabricación de empaques para alimentos, ya que la inclusión de la FS tuvo un efecto significativo sobre algunas propiedades del biomaterial desarrollado, respecto a las que películas sin FS, así:
- Se aumentó la resistencia a la rotura en cerca de un $16,6 \%$.

- Se incrementó la permeabilidad de oxígeno 4 veces.

- El porcentaje de degradación del biopolímero con FS fue del $50,82 \%$ a los 3 meses; lo que se traduce en un 18,71\% mayor degradación [30].

\subsection{Biomateriales laminados con FS}

En investigaciones realizadas por Javier Fernández y Donald Ingber, se desarrolló un biomaterial con gran resistencia y dureza, inspirados en la cutícula externa de algunos insectos. El nuevo material fue llamado Shrilk, ya que fue creado por complejas interacciones entre quitina y FS. La dureza del material superó la de cualquiera de sus dos componentes de forma individual y puede competir con aleaciones de aluminio, con la mitad del peso que éstas últimas; además, la flexibilidad del mismo puede variar de acuerdo a su contenido de agua.

A partir de este biomaterial se crearon multi laminados que tienen 3 capas dobles de Shrilk (películas con fases separadas de quitina y FS). Las propiedades de este nuevo material están relacionadas con el orden y las proporciones de ambos elementos, lo que permite obtener un acabado laminar similar al del plástico. Las propiedades y atributos de Shrilk lo convierten en un material adecuado para reemplazar muchos polímeros sintéticos, lo que permite que sea una gran opción para el desarrollo de empaques, bolsas y pañales biodegradables, entre otros [9].

\section{CONCLUSIONES}

La gran cantidad de residuos sólidos que se generan diariamente a nivel mundial, ha promovido muchas investigaciones que están orientadas al aprovechamiento de residuos agroindustriales y el desarrollo de nuevos materiales que permitan tener un menor impacto ambiental. En el caso de la industria serícola, todos los residuos fibrosos que se generan pueden servir de materia prima para la obtención de FS, un biopolímero con especiales propiedades mecánicas y de biodegradabilidad. La FS puede transformarse en películas, geles, esponjas y otras formas más; que tienen gran aplicación en áreas como biomedicina, farmacología, cosmética, empaques, entre otras. En el sector de empaques, se aprovechan ciertas propiedades fisicoquímicas de la FS, como son su biodegradabilidad, resistencia a la tracción y buena permeabilidad de oxígeno. Gracias a estas características que posee la FS, se han desarrollado diferentes biomateriales para empaques de alimentos, en algunos casos siendo la FS funcionalizada por otros componentes y otra en la que se incluye la FS como componente funcional. En la primera situación, se han realizado desarrollos como sensores 
con nanoestructuras metálicas para evaluar la calidad del alimento o recubrimientos con efecto antimicrobiano para el embutido de cárnicos; mientras que, en el segundo caso, se han dado avances en películas biodegradables, recubrimientos para frutas o nuevos biomateriales como "Shrilk", con propiedades mecánicas mejoradas que puede reemplazar empaques plásticos, entre otros. Todos estos adelantos permiten evidenciar el potencial que tiene la FS en el sector y genera un estímulo para continuar en la búsqueda científica de nuevos materiales biodegradables con FS que tengan potencial aplicación en la industria de empaques de alimentos.

\section{REFERENCIAS}

[1] R. Rollandi, «IC Latinoamérica,» Abril 2012. [En línea]. Available: http://www.ic-latinoamerica. com / descargas / pdf / articulos_interes / 2012-04_ problematica_de_la_gestion.pdf. [Último acceso: $0 \overline{1}$ Junio 2016].

[2] S. M. Yepes, L. Montoya Naranjo y S. F. Orozco, "Valorización de residuos agroindustriales - frutas en Medellín y el sur del valle del aburrá, Colombia.,» Revista Facultad Nacional de Agronomía Medellín, vol. 61, n ${ }^{\circ} 1$, pp. 4422-4431, 2008.

[3] G. Henao y L. Márquez, Aprovechamiento de los residuos sólidos orgánicos en Colombia. Tesis posgrado. Universidad de Antioquia, Medellín, 2008, p. 116.

[4] S. Saval, «Aprovechamiento de residuos agroindustriales: pasado, presente $\mathrm{y}$ futuro,» Biotecnologia, vol. 16, n² 2, pp. 14-46, 2012.

[5] P. Viswanath y K. Nand, "Anaerobic digestion of silk industry wastes,» Bioresource Technolog, vol. 49, pp. 273-276, 1994.

[6] A. Restrepo, C. Álvarez, D. Peláez, C. Salas y O. Rojas, «Comparación de la fibroina de seda obtenida a partir de capullos y subproductos fibrosos,» Presentado en VII Congreso Internacional de Materiales, p. 5, 2013.

[7] J. G. Rouse y M. E. Van Dyke, «A review of keratinbased biomaterials for biomedical applications,» Materials, vol. 3, pp. 999-1014, 2010.

[8] L.-D. Koh, Y. Cheng, C.-P. Teng, Y.-W. Khin, X.-J. Loh, T. Si-Yin y M.-Y. Han, «Structures, mechanical properties and applications of silk fibroin materials,» Progress in Polymer Science, vol. 46, pp. 86-106, 2015.

[9] J. Fernández y D. E. Ingber, «Unexpected strength and toughness in chitosan-fibroin laminates inspired by insect cuticle,» Advanced Materials, vol. 24, no 4, pp. 480484, 2012.

[10] G. L. Robertson, Food packaging: principles and practice, USA: CRC Press, 2013.
[11] G. L. Robertson, «Food packaging,» Encyclopedia of Agriculture and Food Systems, vol. 3, pp. 232-249, 2014.

[12] J. Han, Innovations in Food Packaging, ELSEVIER Academic Press, 2014, pp. 1-12.

[13] A. Campbell, «Modified atmosphere packaging (MAP) of foods and its combination with electron beam processing,» de Electron beam pasteurization and complementary food processing technologies, London, Woodhead Publishing, 2015, p. 185-194.

[14] A. Götz, A. Wani, H.-C. Langowski y J. Wunderlich, «Food Technologies: Aseptic Packaging,» de Encyclopedia of Food Safety, Alemania, Academic Press, 2014, p. 124134.

[15] K. L. Yam, «Intelligent packaging to enhance food safety and quality,» de Emerging Food Packaging Technologies, USA, Woodhead Publishing, 2012, p. 137152.

[16] R. Ahvenainen, «Active and intelligent packaging: An introduction,» de Novel food packaging techniques, Finlandia, Woodhead Publishing, 2003, p. 5-21.

[17] E. Almenar, P. Hernandez-mun, A. Lo, R. Gavara y J. M. Lagaro, «Overview of active polymer-based packaging technologies for food applications,» Food Reviews International, vol. 20, n 4, pp. 357-387, 2004.

[18] Universidad de Valencia, «Tema 9. Química Física de los Polímeros,» Química Física Avanzada. Cuarto Curso, pp. 1-43, 2010.

[19] A. Brody, E. Strupinsky y L. Kline, Active Packaging for food applications, USA: CRC Press, 2002.

[20] C. Padeste y S. Neuhaus, «Functional polymer structures,» de Polymer Micro - and Nanografting, Reino Unido, William Andrew Publishing, 2015, pp. 1-10.

[21] R. Cruz-Morfin, Y. Martínez-Tenorio y A. LópezMalo, «Biopolímeros y su integración con polímeros convencionales como alternativa de empaque de alimentos,» Temas de Selección de Ingeniería de Alimentos, vol. 7, n' 2, pp. 42-52, 2013.

[22] A. K. Mohanty, M. Misra y L. T. Drzal, Natural fibers, biopolymers and biocomposites, Boca Raton: Taylor and Francis Group, 2005.

[23] M. Niaounakis, «Definitions of Terms and Types of Biopolymers,» de Biopolymers: Applications and Trends, Reino Unido, William Andrew Publishing, 2015, pp. 1-90.

[24] S. N. Swain, S. M. Biswal, P. K. Nanda y P. Nayak, «Biodegradable Soy-Based Plastics: Opportunities and Challenges,» Journal of Polymers and the Environment, vol. 12, n 1, pp. 35-43, 2004. 
[25] D. Bello Gil, «Eco-sitio,» Instituto Cubano de Investigaciones de los Derivados de la Caña de Azúcar, 2 Diciembre 2009. [En línea]. Available: http:/ / www.ecositio.com.ar/node/114. [Último acceso: 25 Junio 2016].

[26] D. Gabor y O. Tita, «Biopolymers used in food packaging : a Review,» Acta Universitatis Cibiniensis Series E: Food Technology, vol. XVI, nº 2, pp. 3-19, 2012.

[27] A. González y C. Álvarez, «Soy protein e Poly (lactic acid) bilayer films as biodegradable material for active food packaging,» Food Hydrocolloids, vol. 33, n 2, pp. 289-296, 2013.

[28] P. Cinelli, M. Schmid, E. Bugnicourt, J. Wildner, A. Bazzichi, I. Anguillesi y A. Lazzeri, «Whey protein layer applied on biodegradable packaging film to improve barrier properties while maintaining biodegradability,» Polymer Degradation and Stability, vol. 108, p. 151-157, 2014

[29] P. Fernandez-Saiz, "Chitosan polysaccharide in food packaging applications,» de Multifunctional and Nanoreinforced Polymers for Food Packaging, España, Woodhead Publishing, 2011, p. 571-593.

[30] P. Kuchaiyaphum, W. Punyodom, S. Watanesk y R. Watanesk, "Composition optimization of polyvinyl alcohol/rice starch/silk fibroin-blended films for improving its eco-friendly packaging properties,» Journal of Applied Polymer Science, vol. 119, $\mathrm{n}^{\circ}$ 5, pp. 2614-2620, 2013.

[31] A. Rodríguez, J. Vargas, J. Rodríguez, A. Martínes, F. Lara, M. Ehsan y F. M. Lara, Manual de la sericultura en Hidalgo, México D.F.: Alquimia, 2012, p. 103.

[32] J. Hardy y T. Scheibel, «Composite materials based on silk proteins,» Progress in Polymer Science, vol. 35, $\mathrm{n}^{\circ}$ 9, pp. 1093-1115, 2010.

[33] Y. Yanagi, Y. Kondo y K. Hirabayashi, «Deterioration of silk fabrics and their crystallinity,» Textile Research Journal, vol. 70, $\mathrm{n}^{\mathrm{o}}$ 10, pp. 871-875, 2000.

[34] J. Bernstein, Polymorphism in Molecular Crystal, Oxford: Oxford Science Publications, 2002.

[35] C. Vepari y D. Kaplan, «Silk as a biomaterial,» Progress in Polymer Science, vol. 32, no 8-9, pp. 991-1007, 2007.

[36] J. Magoshi, Y. Magoshi, M. Becker, M. Kato, Z. Han, T. Tanaka, S. I. Inoue y S. Nakamura, «Crystallization of silk fibroin from solution,» Thermochimica Acta, Vols. $\% 1$ de $\% 2352-353$, pp. 165-169, 2000.

[37] O. Rojas, M. I. Briceño y J. Avendaño, «Fundamentos de reología [Internet]. Universidad de los Andes de
Venezuela,» 2012. [En línea]. Available: http:/ / www.firp. ula.ve/archivos/cuadernos/S521C.pdf. [Último acceso: 29 Diciembre 2015].

[38] M. Wagner, «Thermogravimetric analysis,» de Thermal Analysis in Practice, Suiza, Mettler Toledo, 2009, pp. 146-170.

[39] H. García, «Electroforesis en geles de poliacrilamida: fundamentos, actualidad e importancia,» Universo Diagnóstico, vol. 1, n 2, pp. 31-41, 2001.

[40] B. Kundu, N. E. Kurland, S. Bano, C. Patra, F. B. Engel, V. K. Yadavalli y S. C. Kundu, «Silk proteins for biomedical applications: Bioengineering perspectives,» Progress in Polymer Science, vol. 39, n 2, pp. 251-267, 2014.

[41] D. Rockwood, R. Preda, T. Yucel, X. Wang, M. Lovett y D. Kaplan, «Materials fabrication from Bombyx mori silk fibroin,» Nature Protocols, vol. 6, n 10, pp. 16121631, 2011.

[42] S. Aznar-Cervantes, D. Vicente-Cervantes, L. Meseguer-Olmo, J. Cenis y A. Lozano-Pérez, «Influence of the protocol used for fibroin extraction on the mechanical properties and fiber sizes of electrospun silk mats,» Materials Science and Engineering C, vol. 33, $\mathrm{n}^{\circ} 4$, pp. 1945-1950, 2013.

[43] H.-Y. Wang y Y.-Q. Zhang, «Effect of regeneration of liquid silk fibroin on its structure and characterization,» Soft Matter, vol. 9, n 1, pp. 138-145, 2012.

[44] A. Murphy y D. Kaplan, «Biomedical applications of chemically-modified silk fibroin,» Journal of Materials Chemistry, vol. 19, nº 36, pp. 6443-6450, 2009.

[45] J. Wong, H. K. Chan y W. Chrzanowski, «Silk for pharmaceutical and cosmeceutical applications,» de Silk Biomaterials for Tissue Engineering and Regenerative Medicine, Sydney, Woodhead Publishing, 2014, p. 519545 .

[46] H. Tao, M. Brenckle, M. Yang, J. Zhang, L. Mengkun, S. Siebert, R. Averitt, M. Mannoor, M. McAlpine, J. Rogers, D. Kaplan y F. Omenetto, «Silk-based conformal, adhesive, edible food sensors,» Advanced Materials, vol. 24, pp. 1067-1072, 2012.

[47] A. Uçar y D. Keriman, Antimicrobial properties of silk fibroin- carrageenan films incorporating grape seed extract a thesis submitted to in food engineering. Tesis de Maestría, Turquía: İzmir Institute of Technology, 2009.

[48] R. F. Weska, J. Vieira, C. Wellington, G. M. Nogueira y M. M. Beppu, «Effect of freezing methods on the properties of lyophilized porous silk fibroin membranes,» Materials Research, vol. 12, nº 2, pp. 233-237, 2009. 
[49] B. Dhandayuthapani, Y. Yasuhiko, T. Maekawa y D. S. Kumar, «Fabrication and characterization of nanofibrous scaffold developed by electrospinning," Materials Research, vol. 14, n 3, pp. 317-325, 2011.

[50] Y. Baimark, P. Srihanam y Y. Srisuwan, «Praparation of flexible silk fibroin film plasticized with glucose,» Asian Journal of Material Sciences, vol. 1, pp. 29-35, 2009.

[51] Y. Baimark, P. Srihanam y Y. Srisuwam, «Effect of chitosan molecular weights on characteristics of silk fibroin - chitosan blend films,» Current Research in Chemistry, vol. 1, pp. 8-14, 2009.

[52] Y. Ma y K. B. Song, «Physical properties of silk fibroin films treated with various plasticizers,» Food Science and Nutrition, vol. 10, n 2, pp. 187-190, 2005.

[53] K.-J. Ku, Y.-H. Hong y K. B. Song, «Preparation of a silk fibroin film containing catechin and its application,» Food Science and Biotechnology, vol. 17, n 6, pp. 12031206, 2008.

[54] Y. T. Kim, B. Min y K. W. Kim, «General characteristics of packaging materials for food system,» de Innovation in Food Packaging, USA, Academic Press, 2014, pp. 13-35.

[55] S. Torres-Giner, «Electrospun nanofibers for food packaging applications,» de Multifunctional and Nanoreinforced Polymers for Food Packaging, España, Woodhead Publishing, 2011, p. 108-125.

[56] J. Weiss, K. Kanjanapongkul, S. Wongsaulak y T. Yoovidhya, «Electrospun fibers: fabrication, functionalities and potential food industry applications,» de Nanotechnology in the food, beverage and nutraceutical industries, Reino Unido, Woodhead Publishing Limited, 2012, pp. 362-397.

[57] C. Yao, X. Li, T. Song, Y. Li y Y. Pub, «Biodegradable nanofibrous membrane of zein/silk fibroin by electrospinning,» Polymer International, vol. 58, $\mathrm{n}^{\circ} 4$, p. 396-402, 2009.

[58] B. Marelli, M. A. Brenckle, D. L. Kaplan y F. G. Omenetto, «Silk fibroin as edible coating for perishable food preservation,» Nature Publishing Group, pp. 1-11, 2016. 